\title{
Comparison of Clinical Features and Outcome of Dengue Fever and Multisystem Inflammatory Syndrome in Children Associated With COVID-19 (MIS-C)
}

\author{
Gurdeep Singh Dhooria, Shruti Kakkar, Puneet A Pooni, Deepak Bhat, Siddharth Bhargava, Kamal \\ Arora, Karambir Gill, Nancy Goel \\ From Department of Paediatrics, Dayanand Medical College and Hospital, Ludhiana, Punjab.
}

Correspondence to:

Dr Gurdeep Singh Dhooria, Professor, Department of Pediatrics, Dayanand Medical College and Hospital, Ludhiana, Punjab. gurdeep2005123@gmail.com Received: April 17, 2021; Initial review: April 20,2021; Accepted: July 20, 2021.
Objective: To identify clinical and laboratory features that differentiate dengue fever patients from MIS-C patients and determine their outcomes. Methods: This comparative crosssectional study was done at a tertiary care teaching institute. We enrolled all hospitalized children aged 1 month - 18 years and diagnosed with either MIS-C and/or dengue fever according to WHO criteria between June and December, 2020. Clinical and laboratory features and outcomes were recorded on a structured proforma. Results: During the study period 34 cases of MIS-C and 83 cases of Dengue fever were enrolled. Mean age of MIS-C cases (male, $86.3 \%$ ) was 7.89 (4.61) years. MIS-C with shock was seen in 15 cases $(44 \%)$, MIS-C without shock in 17 cases $(50 \%)$ and Kawasaki disease-like presentation in 2 cases $(6 \%)$. Patients of MIS-C were younger as compared to dengue fever $(P=0.002)$. Abdominal pain and erythematous rash were more common in dengue fever. Of the inflammatory markers, mean C reactive protein was higher in MIS-C patients [100.2 (85.1) vs 16.9 (29.3) $\mathrm{mg} / \mathrm{dL}](P<0.001)$. In contrast, serum ferritin levels were higher in dengue fever patients $(P=0.03)$. Mean hospital stay (patient days) was longer in MIS- $C$ compared to dengue fever (8.6 vs 6.5 days; $P=0.014$ ). Conclusions: Clinical and laboratory features can give important clues to differentiate dengue fever and MIS-C and help initiate specific treatment.

Keywords: Diagnosis, Evaluation, Inflammatory markers, Management.

Published online: July 23, 2021; PII:S097475591600355

$\mathrm{M}$ ultisystem inflammatory syndrome in children (MIS-C), an inflammatory condition following severe acute respiratory syndrome coronavirus 2 (SARS-CoV-2), has manifestations similar to toxic shock syndrome or Kawasaki disease [1,2]. Dengue fever can have clinical presentations similar to MIS-C with presence of fever, erythematous rash, vomiting, abdominal pain and development of shock in severe cases. Correct diagnosis and appropriate management are critical to reduce mortality in both the conditions.

We conducted this study to identify clinical and laboratory features that differentiate dengue fever from MIS-C patients admitted in a tertiary care center and determine their outcomes.

\section{METHODS}

In this cross-sectional study, we evaluated all hospitalized children aged 1 month to 18 years diagnosed with MIS-C and/or dengue fever admitted in the department of pediatrics, at our center from June to December, 2020. All patient data were entered in a structured proforma. Patients were followed up till discharge. Written informed consent was taken from all participants and the study was approved by the institutional ethics committee.

All patients with fever for more than three days and fulfilling the World Health Organization (WHO) criteria for MIS-C [1] were included. SARS-CoV-2 infection was diagnosed by nasopharyngeal swab, real time reverse transcription polymerase chain reaction (RT-PCR) for SARS-CoV-2 infection using TRUPCR SARS-CoV-2 (3B BLACK BIO, Kilpest India Ltd) and/or rapid antibody test for SARS-CoV-2 ( $n=50)$ using Elevate Anti-SARS-CoV-2 (IgG and IgM) (Roche Diagnostics GmbH). Additionally, history of contact with a COVID-19 (coronavirus disease 2019) positive patient was also considered positive as per the WHO criteria.

Only confirmed case of dengue fever based on serological evidence by IgM ELISA or by NS1 antigen positivity were included. Patients with dengue infection were classified into two groups viz., dengue with warning signs and severe dengue, according to WHO classi- 
fication [5]. Dengue antibody test for IgM detection was done using an IgM antibody-capture enzyme-linked immunosorbent assay (MAC-ELISA) (PanBio, Standard Diagnostics Inc). Dengue NS1 antigen was detected with the ELISA technique (J Mitra \& Co Pvt Ltd).

Patients were serially monitored clinically and by laboratory parameters and managed as per standard guidelines. We collected demographic data; past medical history, co-morbidities, clinical signs and symptoms, results of imaging, cardiac, and laboratory testing for signs of inflammation (elevated C-reactive protein (CRP), erythrocyte sedimentation rate (ESR), fibrinogen, d-dimer, ferritin, lactic acid dehydrogenase (LDH), or interleukin 6 (IL-6), elevated neutrophils, reduced lymphocytes and low albumin) and organ involvement, at presentation and throughout the hospital stay. The information with respect to need for respiratory and inotropic support, medications like steroids and intravenous immune globulin (IVIG), duration of hospital stay and survival was also collected. Clinical patterns of MIS-C patients including those with or without shock and coronary involvement were also noted. Left ventricle dysfunction was graded on 2D-echo as: normal function ( $\mathrm{EF}>55 \%$ ), mild dysfunction ( $\mathrm{EF} 41-55 \%$ ), moderate dysfunction (EF 31-40\%), and severe dysfunction ( $\mathrm{EF} \leq 30 \%$ ) [6]. The American Heart Association criteria for Kawasaki disease were used [7].

To achieve a power of $80 \%$ and a level of significance of $5 \%$ (two sided), for detecting a true difference of 4 days (7.9-3.8 days) in mean duration of hospital stay between MIS-C and dengue fever cases from previous studies [3,4] assuming a pooled standard deviation of 5 days, minimum sample size of 24 for each group was calculated.

Statistical analysis: Comparison of quantitative variables was done using Student $t$-test and Mann-Whitney $U$ test for independent samples for parametric and nonparametric data, respectively. For comparing categorical data, chi-square test was used. Kaplan-Meier analysis was used to estimate the duration of hospital stay in the three groups, with the end point as time of discharge. Statistical analyses were performed using SPSS version 24.0. $P<0.05$ was considered statistically significant.

\section{RESULTS}

Of the 34 MIS-C cases, MIS-C with shock was seen in 15 (44\%) children, MIS-C without shock in $17(50 \%)$ children and Kawasaki like presentation in two children (6\%). Of the 83 cases of dengue fever, 51(61\%) cases had severe dengue by WHO classification. Mean (SD) age of children with dengue fever was 10.07 (4.43) years compared to MIS-C, 7.18 (4.81) years $(P=0.002)$. MIS-C patients had more frequent symptoms of fever, conjunctival injection, swelling of hand and feet, diarrhea and altered sensorium. Whereas, abdominal pain and erythematous rash were more commonly noted in dengue fever patients (Table I). Clinical bleeding was seen only in dengue fever patients (7\%). Mean (SD) hematocrit was significantly higher in dengue fever compared to MIS-C patients [38.6\% (8.1\%) vs. $29.1 \%(6.9 \%) ; P<0.001]$. Mean platelet count and total leukocyte count was significantly lower in dengue fever compared to MIS-C patients.

Of the inflammatory markers, mean CRP was higher in MIS-C patients than dengue fever patients. Mean IL-6

Table I Clinical Profile, Management and Outcome of Children With MIS-C and Dengue Fever

\begin{tabular}{|c|c|c|}
\hline Group & $\begin{array}{c}M I S-C \\
(n=34)\end{array}$ & $\begin{array}{r}\text { Dengue } \\
(n=83)\end{array}$ \\
\hline \multicolumn{3}{|l|}{$A g e^{a}$} \\
\hline $0-5 y$ & $12(35)$ & $12(14)$ \\
\hline $6-10 y$ & $16(47)$ & $35(42)$ \\
\hline $11-18$ y & $6(18)$ & $36(43)$ \\
\hline Male gender & $28(82)$ & $62(75)$ \\
\hline \multicolumn{3}{|l|}{ Signs/symptoms } \\
\hline Fever at admission ${ }^{b}$ & $34(100)$ & $60(72)$ \\
\hline Diarrhea $^{a}$ & $4(12)$ & 0 \\
\hline Abdominal pain ${ }^{c}$ & $12(35)$ & $47(57)$ \\
\hline Vomiting & $17(50)$ & $57(69)$ \\
\hline Erythematous rash ${ }^{b}$ & $9(26)$ & $55(66)$ \\
\hline Swelling of hand and feet ${ }^{b}$ & $10(29)$ & 0 \\
\hline Respiratory distress & $14(41)$ & $38(46)$ \\
\hline Altered sensorium ${ }^{c}$ & $8(24)$ & $6(7)$ \\
\hline Conjunctival injection $^{b}$ & $7(21)$ & 0 \\
\hline Myalgia $^{b}$ & $6(18)$ & $68(83)$ \\
\hline Signs of capillary leak & $13(38)$ & $36(43)$ \\
\hline Hypotension at admission & $15(44)$ & $33(39)$ \\
\hline \multicolumn{3}{|l|}{ Imaging } \\
\hline USG-moderate ascitis & $10(59)$ & $15(71)$ \\
\hline$X$-ray pleural effusion & $10(29)$ & $28(34)$ \\
\hline LV dysfunction ${ }^{b}$ & $7(21)$ & 0 \\
\hline \multicolumn{3}{|l|}{ Management } \\
\hline Non-invasive ventilation & $6(18)$ & $18(22)$ \\
\hline Mechanical ventilation $^{a}$ & $6(18)$ & $2(2)$ \\
\hline Inotropes & $13(38)$ & $22(27)$ \\
\hline Platelet transfusion $^{c}$ & 0 & $10(12)$ \\
\hline \multicolumn{3}{|l|}{ Patient outcome } \\
\hline PICU admission & $11(32)$ & $21(25)$ \\
\hline Discharged & $31(98)$ & $81(98)$ \\
\hline
\end{tabular}

All values in no. (\%). MIS-C: multi-system inflammatory syndrome in children associated with COVID-19; USG: ultrasonography, IVIG: intravenous Immunoglobulin, LMWH - low molecular weight heparin, PICU: pediatric intensive care unit. Steroids, intravenous immunoglobulin, low molecular weight heparin and aspirin were used in 22, 11,8 and 8 children with MIS-C and none with dengue fever. ${ }^{a} P<0.01 ;{ }^{b} P=0.001 ;{ }^{c} P<0.05$. 
levels, D-dimer and fibrinogen levels were also higher in MIS-C patients. In contrast, mean serum ferritin levels were higher in dengue fever patients. Left ventricular dysfunction was present only in MIS-C patients (Table II and Web Fig 1). Need for mechanical ventilation was more in MIS-C cases as compared to dengue fever cases. Intravenous immunoglobulin (IVIG) infusion, steroids, low molecular weight heparin and aspirin were used only in MIS-C cases (Table I).

Kaplan-Meier survival curve with discharge as end point showed significantly longer duration of hospital stay in MIS-C patients [8.58 days (95\% CI 7.13 - 10.03)] compared to dengue fever patients [6.54 days $(95 \% \mathrm{CI}$ : $5.78-7.21)](P=0.014)$ (Fig. 1).

Repeat 2D-echocardiography was done before discharge in all patients with LV dysfunction/ Kawasaki disease like presentation. Only two children (5.9\%) showed cardiac dysfunction, one case each with mild and moderate dysfunction. Both the cases showed resolution during follow-up.

\section{DISCUSSION}

The study compares the clinical and laboratory differences and outcomes of children hospitalized with MIS-C and dengue fever. Patients with dengue fever were significantly older as compared to MIS-C. Inflammatory

Table II Laboratory Profile and Outcomes of Children With MIS-C and Dengue Fever

\begin{tabular}{|c|c|c|}
\hline Laboratory parameters & $M I S-C(n=34)$ & Dengue $(n=83)$ \\
\hline \multicolumn{3}{|l|}{ Serum values } \\
\hline $\mathrm{CRP}(\mathrm{mg} / \mathrm{L})^{a}$ & $100(85)$ & $17(29)$ \\
\hline Ferritin $(\mathrm{ng} / \mathrm{mL})^{b}$ & $2878(5876)$ & $6136(6600)$ \\
\hline D-Dimer $(\mathrm{ng} / \mathrm{mL})^{b}$ & $1619(1313)$ & 733(291) \\
\hline Interleukin-6 ( pg/mL) & $677(1505)$ & $11(15)$ \\
\hline Fibrinogen $(\mathrm{mg} / \mathrm{dL})^{c}$ & $547(98)$ & $238(121)$ \\
\hline $\operatorname{Hemoglobin}(\mathrm{g} / \mathrm{dL})^{a}$ & $9.7(2.3)$ & $12.9(2.7)$ \\
\hline Leukocyte count $\left(\times 10^{9} / \mathrm{L}\right)^{a}$ & $16.6(12)$ & $7(5.7)$ \\
\hline Platelets $\left(\times 10^{9} / \mathrm{L}\right)^{a}$ & $173.4(134.7)$ & $48.1(42)$ \\
\hline $\operatorname{AST}(\mathrm{U} / \mathrm{L})$ & $524(1633)$ & $641(1359)$ \\
\hline $\operatorname{ALT}(\mathrm{U} / \mathrm{L})$ & $236(569)$ & $277(531)$ \\
\hline $\operatorname{Albumin}(\mathrm{g} / \mathrm{dL})^{b}$ & $3(1.1)$ & $3.5(0.8)$ \\
\hline LV ejection fraction $(\%)$ & $52(13)$ & $60(0)$ \\
\hline PRISM II score & $9(8)$ & $8(6)$ \\
\hline Hospital stay (d) & $8.1(4.1)$ & $6.5(3.3)$ \\
\hline
\end{tabular}

Data prepented as mean (SD). MIS-C: Multi-system inflammatory syndrome in children associated with COVID-19; CRP-C-reactive protein; AST-aspartate transaminase; ALT-alanine transaminase; $L V$ : Left ventricular;PRISM score II-Pediatric risk of mortality score. ${ }^{a} P<0.001,{ }^{b} P<0.05,{ }^{c} P<0.01$.

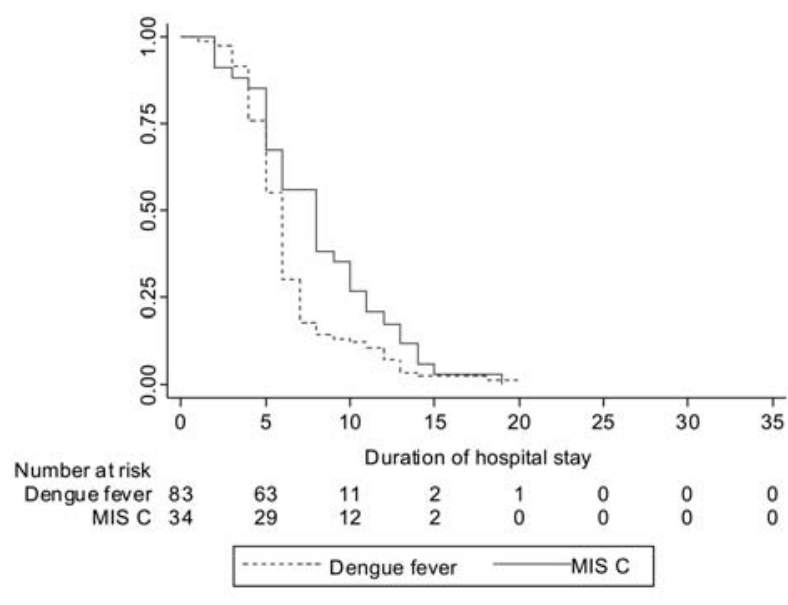

Fig. 1 Kaplan-Mayer graph showing mean duration of hospital stay in children with dengue fever and multisystem inflammatory syndrome in children associated with COVID-19 (MIS-C).

marker levels of CRP, IL-6, D dimer and fibrinogen were significantly higher in MIS-C as compared to dengue fever patients.

All studies on MIS-C have reported hyperinflammatory state as a primary hallmark $[8,9]$. The massive release of inflammatory mediators seen with exaggerated activation of the immune system is similar to cytokine storm syndrome [10]. It has been hypothesized that severe dengue is also caused by a cytokine storm inducing systemic inflammatory effects [11].

Although post-COVID MIS-C can present with lower platelet counts but the severe thrombocytopenia $(<50$ $\mathrm{x} 10^{9} / \mathrm{L}$ ) as seen in dengue fever, is not common [8]. Also, hemoconcentration is uncommon in MIS-C patients, making it an important differentiating feature of dengue fever from MIS-C. Leukopenia followed by thrombocytopenia, capillary leak and hemoconcentration is very classic and pathognomonic of dengue fever.

Cornelia, et al. [12] showed presence of hyperferritinemia could discriminate between dengue and other febrile diseases. Other dengue studies also found an association between increased ferritin levels and severity of disease $[12,13]$. We also found serum ferritin levels to be higher in dengue fever patients than MIS-C patients.

In the present study, most patients requiring invasive ventilation were in MIS-C group (18\%) as compared to dengue patients $(2 \%)$. Other studies have also shown similar results. $[9,14]$.

The differentiation between dengue fever and MIS-C is important in contemporary times because of entirely different management strategy for the two conditions. Dengue fever patients being managed with aggressive 


\section{WHAT THIS STUDY ADDS?}

- We provide clinical and laboratory indicators that can give clues to differentiate dengue fever from MIS-C patients.

fluid management of crystalloids and colloids with inotropic support and platelet transfusions wherever needed. On the other, such aggressive fluid management in MIS-C patients would be detrimental in patients with cardiac dysfunction that is often present in MIS-C patients with shock. Moreover, vital role of intravenous immunoglobulin and steroids in management of MIS-C patients can never be overemphasized.

The study has few limitations. Firstly, the study is an experience from a single center. The diagnosis of dengue fever was based on serology in one third of cases. Studies have shown that COVID-19 cases may be misdiagnosed as dengue fever when relying on DENV IgM, which can remain positive months after COVID-19 infection [15].

To conclude, the presence of conjunctival injection, swelling of hand and feet, diarrhea, and altered sensorium in a febrile child with laboratory evidence of hyperinflammation (highly raised CRP, leukocytosis, raised Ddimers are pointers more in favor of MIS-C. Whereas, vomiting, myalgia and erythematous rash along with hyperferritinemia, hemoconcentration, leukopenia and severe thrombocytopenia are more common in dengue fever patients.

Acknowledgements: Namita Bansal, Statistician for analyzing the data and for preparation of tables and graphs. Dr Jatinder Goraya for his advice and support.

Note: Additional material related to this study is available with the online version at $w w w$.indianpediatrics.net

Ethics clearance: Institutional ethics committee; No: DMCH/ R\&D/2020/168 dated November 09, 2020.

Contributors: GSD, PAP: conceived and designed the study: SK, $\mathrm{NG}, \mathrm{KG}$ : recruited the subjects, collected the data; KA, SB, GSD: literature review, initial draft of manuscript; PAP, DB, GSD: contributed to manuscript writing; and PAP, DB, GSD: finalized the manuscript. All authors approved the manuscript submitted.

Funding: None; Competing interests: None stated.

\section{REFERENCES}

1. Multisystem inflammatory syndrome in children and adolescents with COVID-19. Scientific brief: World Health Organization. 15 May 2020. Accessed April 15, 2021. Available from: https: //www.who.int/publications-detail/ multisysteminflammatory-syndrome-in-children-andadole scents-with-covid19

2. Alsaied T, Tremoulet AH, Burns JC, et al. Review of cardiac involvement in multisystem inflammatory syndrome in children. Circulation. 2021;143:78-88.

3. Ahmed M, Advani S, Moreira A, et al. Multisystem inflammatory syndrome in children: A systematic review. E Clin Med. 2020;26:100527.

4. Mishra S, Ramanathan R, Agarwalla SK. Clinical profile of dengue fever in children: A study from southern Odisha, India. Scientifica (Cairo). 2016;2016:6391594.

5. World Health Organization. Dengue Guidelines for diagnosis, treatment, prevention and control: New edition

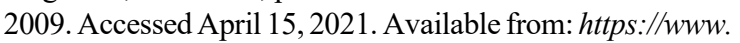
who.int/rpc/guidelines/9789241547871/en/

6. Tissot C, Singh Y, Sekarski N. Echocardiographic evaluation of ventricular function-for the neonatologist and pediatric intensivist. Front Pediatr. 2018;6:79.

7. McCrindle BW, Rowley AH, Newburger JW, et al; American Heart Association Rheumatic Fever, Endocarditis, and Kawasaki Disease Committee of the Council on Cardiovascular Disease in the Young; Diagnosis, Treatment, and Long-Term Management of Kawasaki Disease: A Scientific Statement for Health Professionals From the American Heart Association. Circulation. 2017;135:e927-e999.

8. Feldstein LR, Rose EB, Horwitz SM, et al. Overcoming COVID-19 Investigators; CDC COVID-19 response team. Multisystem inflammatory syndrome in U.S. children and adolescents. N Engl J Med. 2020;23:334-46.

9. Rowley AH. Understanding SARS-CoV-2-related multisystem inflammatory syndrome in children. Nat Rev Immunol. 2020;20:453-54.

10. Alunno A, Carubbi F, Rodríguez-Carrio J. Storm, typhoon, cyclone or hurricane in patients with COVID-19? Beware of the same storm that has a different origin. RMD Open. 2020;6:e001295.

11. Srikiatkhachorn A, Mathew A, Rothman AL. Immunemediated cytokine storm and its role in severe dengue. SeminImmunopathol. 2017;39:563-74.

12. van de Weg CA, Huits RM, Pannuti CS, et al. Hyperferritinaemia in dengue virus infected patients is associated with immune activation and coagulation disturbances. PLoSNegl Trop Dis. 2014;8:e3214.

13. Chaiyaratana W, Chuansumrit A, Atamasirikul K, Tangnararatchakit K. Serum ferritin levels in children with dengue infection. Southeast Asian J Trop Med Public Health. 2008;39:832-36.

14. Jain S, Sen S, Lakshmivenkateshiah S, et al. Multisystem inflammatory syndrome in children with COVID-19 in Mumbai, India. Indian Pediatr. 2020;57:1015-19.

15. Lokida D, Lukman N, Salim G, et al. Diagnosis of COVID19 in a dengue-endemic area. Am J Trop Med Hyg. 2020;103:1220-22. 